\title{
Congestion Management for Urban EV Charging Systems
}

\author{
Emmanouil S. Rigas*, Sarvapali D. Ramchurn ${ }^{\dagger}$, Nick Bassiliades ${ }^{*}$, George Koutitas ${ }^{\ddagger}$ \\ *Department of Informatics, Aristotle University of Thessaloniki, 54124, Thessaloniki, Greece \\ \{erigas, nbassili\}@csd.auth.gr \\ $\dagger$ Electronics and Computer Science, University of Southampton, Southampton, SO17 1BJ, UK \\ sdr1@soton.ac.uk \\ $\ddagger$ School of Science and Technology, International Hellenic University, Greece \\ g.koutitas@ihu.edu.gr
}

\begin{abstract}
We consider the problem of managing Electric Vehicle (EV) charging at charging points in a city to ensure that the load on the charging points remains within the desired limits while minimizing the inconvenience to EV owners. We develop solutions that treat charging points and EV users as self-interested agents that aim to maximize their profit and minimize the impact on their schedule. In particular, we propose variants of a decentralised and dynamic approach as well as an optimal centralised static approach. We evaluated these solutions in a real setting based on the road network and the location of parking garages of a UK city and show that the optimal centralised (nondynamic) solution manages the congestion the best but does not scale well, while the decentralised solutions scale to thousands of agents.
\end{abstract}

\section{INTRODUCTION}

With dwindling fossil fuels, and the increasingly negative impact of climate change on society, several countries have instigated national plans to reduce carbon emissions [1]. In particular, the electrification of transport is one of the main pathways to achieve significant reductions in CO2 emissions. However, the ageing grid infrastructure in most countries stands in the way of the wider deployment of EVs as they may result in unpredictable, and significant loads on the grid, that may, in turn, cause imbalances, leading to blackouts or brownouts.

In this paper we study an urban EV charging setting where EVs drive across a city converge to parking stations in the centre and need to charge. The EVs are self-interested agents that want to charge their vehicle achieving the maximum wellfare, while from a system's perspective, the balanced distribution of the EVs accross the charging points is the objective. Hence, it is crucial to leverage advances in decentralised control and mechanism design to coordinate demand and supply to mitigate the impact on the grid.

In order to allocate the EVs to charging points we propose two solutions, a decentralized and a centralized. In the decentralized solution the EVs arrive dynamically at the system over time, and the decision on the charging point to charge needs to be taken in real time and locally. In this case, we have used three different strategies for online allocation of time slots at the charging points namely Naive, Random and Smart. In the centralized static optimal solution we have full knowledge of the arrival time and the preferences of the EVs in advance and is being used for benchmarking reasons. In both solutions, dynamic pricing techniques are used in order to manage the traffic while, in all cases, the cost function of the EVs is the same and all charging points follow the same pricing strategy.

In this field, limited related work exists. Gerding et al [2] present a similar setting, where EVs want to charge in charging points fullfilling their objectives, but the presented solution is abstract and is tested on a simple and unrealistic setting where the charging points are located in houses and they have only one charging slot each.

Research undertaken in the area of load management regarding EVs charging, includes Gerding et al. [3] who have designed an online auction protocol, in which EV drivers are using agents in order to bid for power. Stein et al. [4] improve this protocol as they introduce the notion of pre-commitment keeping though a flexibility regarding when the charging takes place and at what rate. However, they study a one-sided setting with a single, fixed charging point. In addition, Caramanis et al. [5] have studied the management of EV load within a market based Electric Power System Control Area and suggest a hierarchical decision making methodology. Finally, Eisner et al. [6] and Storandt et al. [7] have addressed the problem of routing of EVs in an energy efficient way, but they do not consider the allocation of EVs to charging points nor the cost in terms of comfort to the driver.

Our contributions to the state of the art are:

- We propose an optimal centralized solution to solve the problem of matching EVs to charging points, while managing congestion in the optimal way, assuming we could have full knowledge of arrival time and preferences of agents in advance. 
- We propose a decentralized solution with a charging point selection algorithm which matches EVs to charging points trying to maximize utility of each agent.

- Regarding the decentralized solution, we propose three different time point allocation strategies, considering when each EV will charge in the available time window, which combined with the also proposed dynamic pricing function, minimize the cost for the agents while congestion in charging points (number of EVs charging together) is kept balanced.

- In order to test our setting, we have set a simulation based on the road network of the city of Southampton in the UK as taken by Google maps ${ }^{1}$ and we locate the charging points in real parking garages around the city using data from Southampton Romanse ${ }^{2}$. We have run a number of simulations with multiple charging points and charging slots within, as well as a fleet of EVs starting from 10 and going up to 2000 .

The rest of this paper is structured as follows. Section 2 presents our model of the system, the agents that are being used and the decision making algorithm. Section 3 analyzes the different slot allocation mechanisms as well as the pricing function. Moreover, section 4 details our optimal centralized solution and section 5 describes the experiments that we made in order to test the system's behaviour. Finally, section 6 concludes.

\section{Problem Definition}

EVs arrive dynamically in the system over time and need to charge at the charging points. We assume that each EV driver has her own agent that evaluates the different available options and suggests a charging point to go to, based on the preferences given as input by the driver. This agent could, in a real scenario, reside on the navigation system of the car. Each EV has its own location and properties as described in the following paragraph. On the other hand, the charging points, that each one of them is owned by a different company, want to serve as many clients as possible in order to maximize their profit. Whereas the mechanism's objective is to keep a balance in the traffic of the system while the objectives of the two types of agents are met. In this section we present our model of the agents and the algorithm to compute the best charging point to go to.

\section{A. Agents}

In our model, we define a set of locations $l_{1}, l_{2}, . ., l_{n} \in L$, $L=L_{p} \bigcup L_{\bar{p}}$ that can either be charging points or not. Based on this, we define a transport network as a graph $G=(E, L)$ with nodes $L$ and edges $E$, where $e \in E$ represents the roads and $l \in L$ represents the junctions of the road network. We define discrete time points $t \in T$,

\footnotetext{
${ }^{1}$ http://maps.google.co.uk/

${ }^{2}$ http://www.southampton.romanse.org.uk
}

$T \subseteq N$, while the time is global for the system and the same for all agents. We denote the set of EVs as $i_{1}, i_{2}, \ldots, i_{n} \in I$, where an EV $i$ has a discharge rate $d_{i} \in \Re_{0}^{+}$, a battery capacity $\bar{b}_{i} \in \Re_{0}^{+}(\mathrm{KWh})$, and a battery level at time $t, b_{i t} \in \Re_{0}^{+}, t \in T . \forall l \in L_{p}$ there is a number of charging slots $s_{1}, s_{2}, \ldots, s_{n} \in S_{l}$ and each slot is available at a certain time, $t \in T$. We define an allocation matrix $n_{j t i} \in\{0,1\}$ to represent whether a charging slot at charging point $l_{j}$ is occupied or not at time point $t \in T$ by an agent $i$.

Each EV $i$ departs from the source $l_{i}^{\text {start }}$ at time $t_{i}^{\text {start }} \in T$ and wants to travel to destination $l_{i}^{\text {end }}$ where it needs to park for time $\tau_{i}^{\text {parking }} \in N$ where $\tau \in Z$ is a number of time points $t$. Every EV is available to charge between $t_{i j}^{\text {arrival }}=t_{i}^{\text {start }}+\tau_{i j}^{\text {driving }}$ and $t_{i j}^{\text {departure }}=$ $t_{i}^{\text {start }}+\tau_{i j}^{\text {driving }}+t_{i}^{\text {parking }}$. Each vehicle, also needs to charge a specific amount of energy $b_{i j}^{\text {charge }}$ at the charging point $l_{j}$. Each charging point $l_{j}$ has a charging rate, $c_{j} \in \Re_{0}^{+}$which depends on the charging power assigned by the charging point to the EV.

Given a pair of locations $\left(l_{i}^{\text {start }}, l_{i}^{\text {end }}\right)$ and the transport network $G$, the shortest route, $r_{i j} \in R_{i j}$ from the source to the destination is calculated using Dijsktra's algorithm. Similarly, the shortest routes $r_{i j} \in R_{i j}$ from the sources to all charging points $l_{p} \in L$ are calculated as well. Every route $r_{i j}$ has a distance $\delta: R_{i j} \rightarrow \Re$ measured in miles, a time to travel $\tau_{i j}^{d r i v i n g}: R_{i j} \rightarrow Z$, and an amount of energy needed, $E_{\text {needed }}\left(r_{i j}, d_{i}\right)$. Based on this information, the charging points with free slots within the time window the EV will be available to charge, are calculated using function $B\left(S_{j}, t_{i j}^{\text {arrival }}, t_{i j}^{\text {departure }}\right)$.

Based on slot availability and on the ability of an EV to reach a charging point with its initial battery level, a set of valid charging points $\Gamma \subseteq L_{p}$ is defined.

In order to measure the total cost for an EV, we consider two types of cost. The first is the price that the agent will pay at the charging point in order to charge. The second is a set of time-based costs. In particular, we consider the time to drive to the charging point, $\tau_{i j}^{d r i v i n g} \in Z$, the time to walk from the charging point to the final destination, $\tau_{i j}^{\text {walking }} \in Z$, and the time it will need to charge in order to get the battery level needed, $\tau_{i j}^{\text {charging }} \in Z$. It has to be mentioned that we differentiate between charging time and parking time since the EV will not necessarily charge for all the time that it is parked. Moreover, as discussed in Section III, the driver pays for every time slot during which her car is charging, so the fewer the number time points she uses the lower the monetary cost incurred. We assume that each time point spent by the car agent worths one monetary unit. Then, using the function $\omega_{i, j}: R_{i j} \rightarrow \Re_{0}^{+}$ the cost for each route is calculated. This cost function that balances time against money is defined for agent $i$ as:

$$
\omega_{i}\left(r_{i j}\right)=k \times \omega_{i}^{T}\left(r_{i j}\right)+(1-k) \times p_{i j}\left(r_{i j}\right)
$$

where $k \in[0,1]$ is a parameter that is used to trade-off 
between time and money and $w_{i}^{T}\left(r_{i j}\right)$ is the time cost computed as follows:

$$
\omega_{i}^{T}\left(r_{i j}\right)=w_{1} \times \tau_{i j}^{\text {charging }}+w_{2} \times \tau_{i j}^{\text {driving }}+w_{3} \times \tau_{i j}^{\text {walking }}
$$

where $\sum_{i=1}^{3} w_{i}=1$. Weights $w_{1}, w_{2}, w_{3}$ depend on the preferences of the agent.

\section{B. Charging Point Selection Algorithm}

In this section, we present the algorithm run by each EV agent to compute the best charging point to go to based on the estimated travel time (using the shortest-path) to all charging points, its current battery level, and the cost to charge at each charging point. While the source and the destination of the journey, as well as the current battery level are provided by the user, the cost of the electricity is provided by the charging points. Different cost policies are presented in the next section.

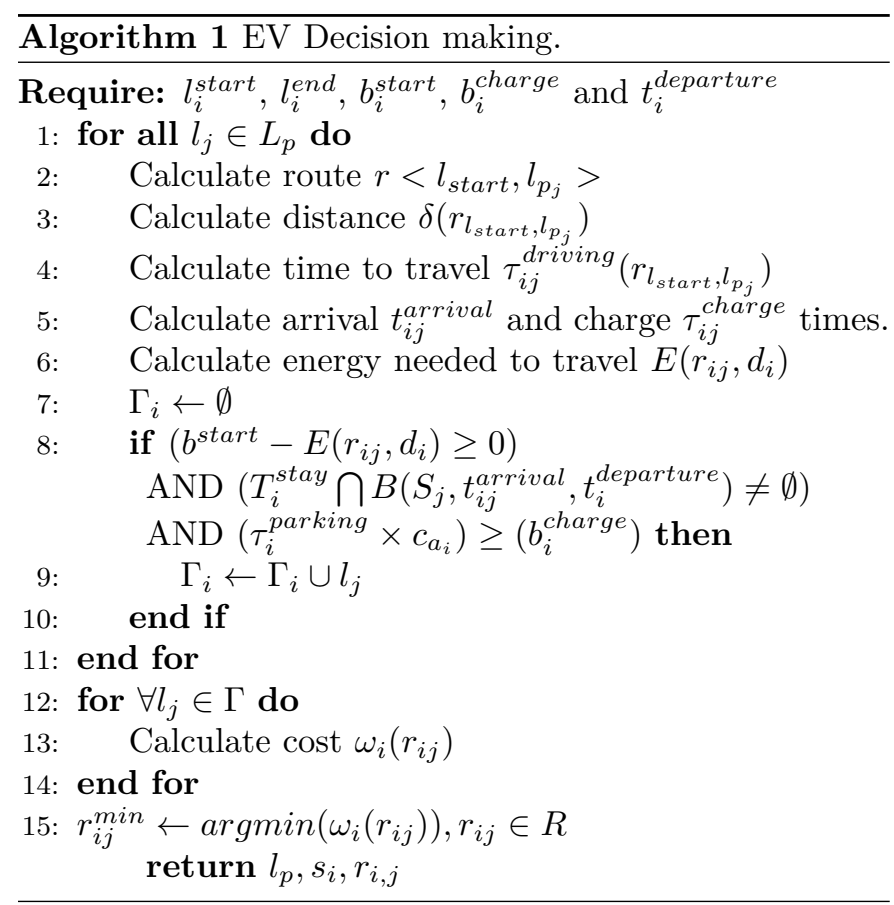

The algorithm takes as input the start and end point of the journey, the battery level before the start of the journey, the amount of energy the EV wants to charge and the desired time of departure. The algorithm calculates the shortest route, distance, time, and energy needed to drive to every charging point (lines 2-6). Then, if the battery level at the beginning of the journey is enough to reach a given charging point, a slot is available for the time period the driver needs to charge, and the charging rate is enough to meet the charging time deadlines, (line 8) the charging point is added to the set of feasible charging points (line 9). Following this, the cost of every option is calculated and added in a set (lines 12-14). After that, the route and, therefore, the charging point, that minimizes the cost is selected (line 15). Finally, the route, the charging point and the slot that the EV is going to charge at, are returned as output by the algorithm.
It has to be mentioned, that if we knew every car's properties that will arrive in the future, and could control all the charging points, we could find the optimal solution (see Section V). However, as we show later, even if this was possible, the solution would not scale to more than a few tens of agents because of high complexity of the optimization algorithm. Rather, we adopt a more realistic scenario that charging points will be individually run by a company that will aim to maximize profit while minimizing congestions, without knowledge of the cars' individual properties a priori. Hence, to develop a scalable online scheduling approach to the management of EVs in an urban environment, in the next secton, we present pricing and charge allocation policies to achieve this.

\section{Calculating Electricity Price}

Now, assuming each EV uses the algorithm presented in the previous section, we turn to the problem of controlling a population of EVs in terms of the charging points they will choose by using a pricing strategy to minimize congestion and maximize profit by increasing or decreasing the price with the number of EVs charging at each time point. Specifically, three pricing strategies have been used namely, Naive, Random and Smart. Crucially, these three strategies present trade-offs in terms of complexity of implementation, congestion generated, and profit generated. Following these, in the next section we present the optimal solution that aims to maximize social welfare (i.e., not only the charging points' profits but also minimize the cost to the EVs and balance congestion). This is the solution against which we will benchmark our pricing heuristics. All strategies use the same price function (3). This is a linear function that is measured as the amount of EVs that charge at each charging point all time points the EV will use to charge. Moreover, all strategies calculate a set of time points $T_{c} \in T$ that the $\mathrm{EV}$ will charge at.

\section{A. Naive}

The simplest way to define the set $T_{i j}^{\text {charge }}$, is to find the first $\tau_{i j}^{\text {charging }}$ time points where free charging slots exist, $\sum_{t}\left(n_{i t j}\right) \leq s_{j}$, between the time of arrival, $t_{i j}^{\text {arrival }}$ and time of departure $t_{i j}^{\text {departure }}$. If charging point $j$ is selected, the time points $t \in T_{i j}^{\text {charge }}$ are booked for agent $i$ and the price is calculated using the folowing equation.

$$
p_{i j}=\forall t \in T_{i j}^{\text {charge }} \sum_{t} \sum_{i}\left(n_{j t i}\right)
$$

\section{B. Random}

This strategy defines the set $T_{i j}^{\text {charge }}$ by randomly selecting $\tau_{i j}^{\text {charging }}$ time points with free charging slots, $\sum_{t}\left(n_{i t j}\right) \leq s_{j}$, between the time of arrival, $t_{i j}^{\text {arrival }}$ and time of departure $t_{i j}^{\text {departure }}$. If charging point $j$ is selected, the time points $t \in T_{i j}^{\text {charge }}$ are booked for agent $i$ and the price is calculated using Equation 3. 


\section{Smart}

This strategy finds, for every charging point $j$, the feasible $\left(\sum\left(n_{i t j}\right)<s_{j}\right)$ time point $t \geq t_{i j}^{\text {arrival }}$ that is the least congested, and therefore is assigned the lowest, and adds to the set $T_{i j}^{\text {charge }}$ this time point and the next $\tau_{i j}^{\text {charging }}-1$ feasible charging points. It has to be mentioned that when selecting the best time point to start, the strategy checks if the $\mathrm{EV}$ will manage to get the battery needed in the remaining feasible time points before the time of departure, $t_{i j}^{\text {departure }}$. If charging point $j$ is selected, the time points $t \in T_{i j}^{\text {charge }}$ are booked for agent $i$ and the price is calculated using Equation 3.

\section{Optimal Solution}

In this section we present a centralized, static, optimal Mixed Integer Quadratic Programming (MIQP) formulation of the problem (solved using IBM ILOG CPLEX), which is compared to the decentralized solution. The aim is to find the optimal charging location and charging times for the EVs, taking into consideration the objectives of the agents regarding battery level after charging and the arrival and departure times, in order to have the minimum overall cost, as described in Equation (4), and the best congestion management.

This solution tries to minimize the objective function (4), which contains the time to charge $t_{i j}^{\text {charge }}$ at charging point $j$, the time to drive $\tau_{i j}^{\text {driving }}$ to charging point $j$, the time to walk $t_{i j}^{\text {walking }}$ to the final destination and the price to pay $p_{i j}$ in order the agent to have the maximum utility. Notice that, in contrast to the decentralized solution where the price is calculated at the time of arrival of each EV to the system, in the offline optimal solution, the price is calculated after the allocation of all EVs as we have full knowledge of the traffic and the objectives of the drivers in advance. Moreover, this price is the same for all vehicles charging at a particular time point $t$ (e.g. If at a particular charging point 4 charging slots exist, and at one time point 3 of them are in use, each $\mathrm{EV}$ will pay price 3 , so alltogether will pay $3 * 3=3^{2}$ ).

In our formulation we define two decision variables. The first one is $y_{i j} \in\{0,1\}$ and indicates whether an agent $i$ charges at charging point $j$ and the second one is $n_{j t i} \in$ $\{0,1\}$ and indicates whether agent $i$ is charging at time point $t$ at charging point $j$.

\section{Objective function:}

$$
\sum_{i \in I} \sum_{r_{i j} \in R_{i j}^{\alpha}}\left(y_{i j} \times \omega_{i}^{T}\left(r_{i j}\right)\right)+\sum_{j} \sum_{t}\left(\sum_{i} n_{j t i}\right)^{2}
$$

\section{Constraints:}

$$
\begin{gathered}
\sum_{r_{i j} \in R_{i j}^{\alpha}}\left(y_{i j}^{\alpha} \leq 1\right), y_{i j} \in\{0,1\} \\
\forall_{i} \forall_{j}, b_{i j}^{\text {end }}=b_{i j}^{\text {charge }} \times y_{i j} \\
\forall_{i} \forall_{j}, b_{i j}^{\text {end }} \leq c_{j} \times \tau_{i j}^{\text {parking }} \times y_{i j} \\
\forall_{j} \forall_{t}, \sum_{i}\left(n_{j t i}\right) \leq s_{j}
\end{gathered}
$$

$$
\forall j \forall i \forall t, t \geq t_{i j}^{\text {arrival }} \text { and } t \leq t_{i j}^{\text {departure }}, \sum_{t} n_{j t i}=\tau_{i j}^{\text {charging }} \times y_{i j}
$$

Explaining the above formulation, EV $i$ will charge at, at most one charging point $j$ (Constraint 5). The main objective of each agent is, after charging at the selected charging point, to get the amount of energy needed. The battery for agent $i$ when it leaves from charging point $j$ $\left(b_{i j}^{e n d}\right)$ must be greater than or equal to the required energy $b_{i j}^{\text {charge }}$ (Constraint 6 ). Moreover, each charging point has a charging rate with which it charges the EVs. The energy that agent $i$ charges at charging point $j$ must be less or equal to the charging rate $c_{j}$ of the charging point times the time that it charges $\tau_{i j}^{\text {parking }}$ (Constraint 7 ).

Every charging point $j$ has a maximum capacity $s_{j}$ which must not be exceeded. Therefore, constraint (8) guarantees that for every charging point $j$ the sum of EVs $i$ that charge at each time point $t$ does not exceed the total capacity of the charging point. Moreover, as constraint (9) guarantees, the sum of all time points that a charging point $j$ is occupied by an agent $i$ must be equal to the time that agent will be charging at the charging point.

\section{Evaluation}

The performance of our pricing strategies depends on the number of EVs, the time they need to charge, and the number of charging points and charging slots. Hence, it is crucial to evaluate them on realistic scenarios and compare them against the optimal (though not scalable, as we show) approach. In order to do so, we run a number of experiments. In this section we present only two of them due to space limitations.. The first is a setting with 10 - 60 electric vehicles where we test the online pricing strategies against the static optimal mechanism. The second experiment is a setting with 200 - 2000 EVs where we test the scalability of our decentralized solution.

In order to evaluate our strategies, three metrics were used. The first is the total monetary cost the EVs need to pay. The second is the sum of squares of the number of EVs that are served at each charging point. This metric (referred to as Parking Balance $(\mathrm{PB})$ ) is important as it portrays how balanced the charging point selection is and how equally distributed the EVs are, as this plays a crucial role in avoiding long waiting queues in certain charging points.

Finally, the third metric is the sum of squares of the number of EVs that charge at each time point at each charging point. This metric (referred to as Charging Balance $(\mathrm{CB}))$ portrays how well balanced the EVs are within each charging point in terms of how many EVs charge simultaneously. Managing the number of EVs charging simultaneously at a charging point is very important in order to avoid peeks in energy demand. This is important for both the consumers of the energy (the charging points) and the producers of the energy, as demand can be leveled and unexpected needs for high volumes of energy avoided. 


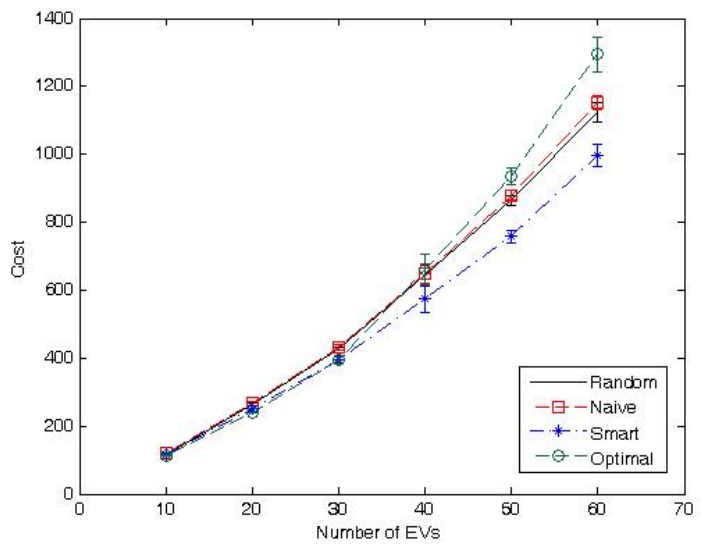

Figure 1. Average Cost of All EVs

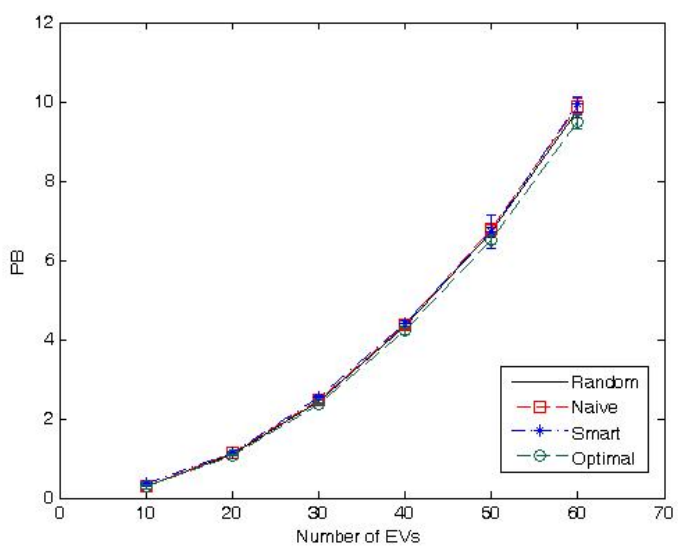

Figure 2. Parking Balance Dynamic - Optimal

It has to be mentioned that, as described in Section 2, a number of battery and charger types exist [8], but in this evaluation, without loss of generality, we assume all EVs are using the same battery type and all charging points the same charger type.

\section{A. Decentralized Solution VS Centralized Solution}

In order to test the pricing strategies of the decentralized solution against the centralized solution we set a simulation experiment where a number of EVs $10 \leq n \leq 60$ arriving in the system have an arrival time $t_{i}^{\text {start }}$ drawn from a normal distribution $\mathrm{N}(65,65)$, parking time $\tau_{i j}^{\text {parking }}$ drawn from a normal distribution $\mathrm{N}(48,8)$, battery needed $b_{i j}^{\text {charge }}$ drawn from a normal distribution $\mathrm{N}(64,8)$ and range $b_{i}^{\text {start }}$ drawn from a normal distribution $\mathrm{N}(20,12)$. Totaly 6 charging points exist with 3 charging slots each.

In terms of PB (Table I and Figure 2), the Naive and Random strategies of the decentralized mechanism are very close to the optimal mechanism, whereas the smart strategy is relatively worse. The poorer performance of the Smart strategy can be attributed to the fact that it searches for the best price. Because of this, it can serve more EVs in a specific charging point without increasing the cost, and therefore, the charging points that are in the centre of the graph, therefore closer to more destinations, get more congested than others.

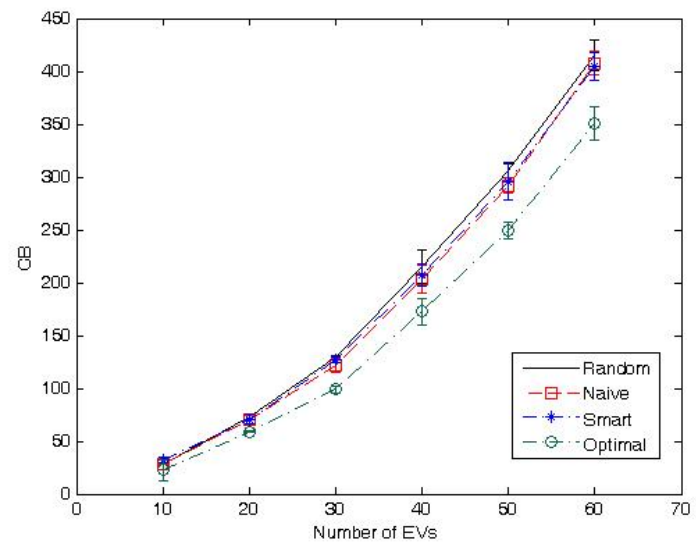

Figure 3. Charging Balance Dynamic - Optimal

In terms of CB (Table I and Figure 3), the optimal solution is, as expected, the best. The Naive strategy achieves better performance compared to both Random and Smart strategies, whereas Smart is better than Random. As it will become clear in the next experiment though, in terms of $\mathrm{PB}$ and $\mathrm{CB}$, the relative performance of dynamic EV charging management mechanism variations change significantly when the number of cars scales up to the thousands.

A straight comparison between the three variants of the decentralized mechanism and the optimal - centralized one in terms of cost cannot be done. This is because the decentralized mechanism calculates the price that each EV will pay based on the EVs that already exist in the system (i.e. incrementally), while the centralized mechanism calculates the price based on the total amount of EVs. So, the dynamic mechanism favors the EVs as they pay less, while the static one favors the charging points as they charge having full knowledge of the future traffic and , therefore, can have an increased revenue. Figure 1 shows how total cost changes on the number of EVs.

Table I

RELATIVE PERFORMANCE OF DYNAMIC SOLUtions VS OPTIMAL

\begin{tabular}{|c|c|c|c|}
\hline & \multicolumn{2}{|c|}{ Number of EVs } \\
\hline & & 30 & 60 \\
\hline \multirow{3}{*}{ Cost } & Naive Vs Optimal & $9.42 \%$ & $-10.96 \%$ \\
\hline & Random Vs Optimal & $8.71 \%$ & $-13.17 \%$ \\
\hline & Smart Vs Optimal & $0.59 \%$ & $-26.17 \%$ \\
\hline \multirow{3}{*}{ PB } & Naive Vs Optimal & $3.36 \%$ & $4.21 \%$ \\
\hline & Random Vs Optimal & $2.1 \%$ & $3.05 \%$ \\
\hline & Smart Vs Optimal & $6.3 \%$ & $4.74 \%$ \\
\hline \multirow{3}{*}{ CB } & Naive Vs Optimal & $22.9 \%$ & $16.31 \%$ \\
\hline & Random Vs Optimal & $29.29 \%$ & $18.45 \%$ \\
\hline & Smart Vs Optimal & $27.9 \%$ & $15.4 \%$ \\
\hline
\end{tabular}

\section{B. Scaling up the Decentralized Solution}

In order to evaluate the scalability of the decentralized mechanism and to compare the three pricing strategies we used a more realistic scenario with a large number of EVs. In particular, $200 \leq n \leq 2000$ arrive in the system at random times $t$ drawn from $\mathrm{N}(200,200)$. We draw parking time, battery needed and range from normal distributions as defined in the previous subsection. Moreover, in total 11 charging points exist and each one has 12 charging slots. 


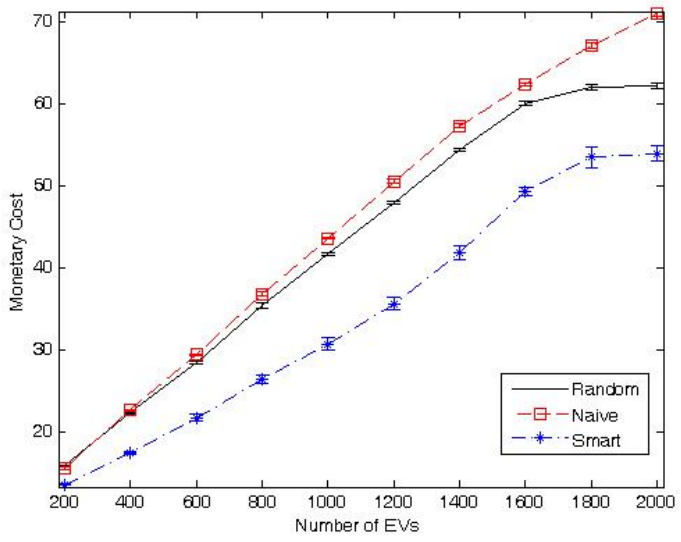

Figure 4. Average Cost of All Serviced EVs

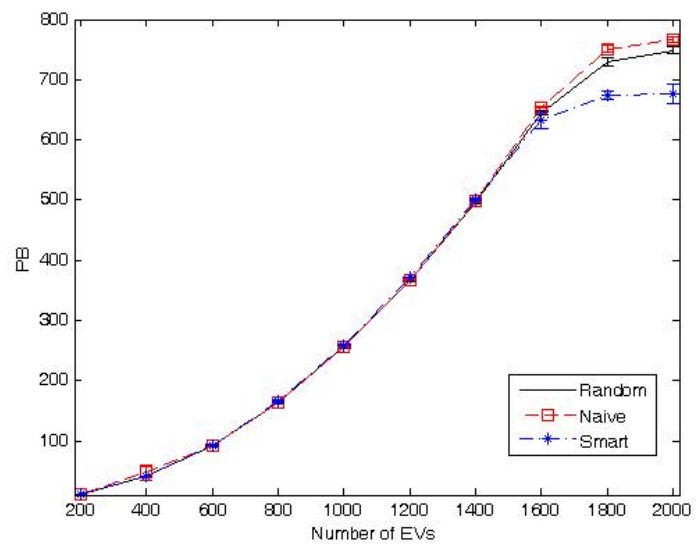

Figure 5. Parking Balance

Table II

Smart and Random Strategies Compared Against Naive

\begin{tabular}{|c|c|c|c|c|}
\hline & \multicolumn{3}{|c|}{ Number of EVs } \\
\hline & & 200 & 1000 & 2000 \\
\hline \multirow[t]{2}{*}{ Cost } & Random Vs Naive & $1.6 \%$ & $-4.35 \%$ & $-15.19 \%$ \\
\hline & Smart Vs Naive & $-13.13 \%$ & $-29.45 \%$ & $-26.43 \%$ \\
\hline \multirow[t]{2}{*}{ PB } & Random Vs Naive & $-0.38 \%$ & $-0.36 \%$ & $-2.33 \%$ \\
\hline & Smart Vs Naive & $1.36 \%$ & $1.24 \%$ & $-11.94 \%$ \\
\hline \multirow[t]{2}{*}{ CB } & Random Vs Naive & $11.39 \%$ & $1.06 \%$ & $-1.66 \%$ \\
\hline & Smart Vs Naive & $8.2 \%$ & $2.12 \%$ & -10.04 \\
\hline
\end{tabular}

In terms of Monetary Cost, the Smart strategy is superior for all numbers of EVs (Table II) and (Figure 4). Moreover, the difference between the Smart strategy and the others increases as the number of EVs grows. On the other hand, Random strategy is worse than Naive when the number of cars is small, around 200, but as the number of cars increases it quickly gets better than the naive.

In terms of $\mathrm{PB}$, the three strategies are close to each other for numbers of EVs below 1400 (Table II). Beyond this point, the congestion in the system increases and the Smart strategy is able to better manage this (see Figure 5), while Naive and Random have similar performance. In terms of charging balance, the image that we get is similar to the parking balance.

Notice that due the large number of EVs, beyond 1600 EVs some of them are not serviced. In more detail, for 2000 EVs the Naive strategy leaves the $26.4 \%$ of EVs unserviced, the random leaves the $13.63 \%$ of the EVs unserviced, whereas the smart leaves only the $5.86 \%$ unserviced. Beyond $1800 \mathrm{EVs}$, where the charging points reach the limits in terms of capacity, the Smart and Random strategies tend to level, while the Naive gets even worse as it services even fewer EVs and at a higher price.

\section{Conclusions}

We propose a decentralized allocation mechanism for the problem of EV charging management in smart city environment that provides load balancing and cost savings even for a large number of EVs. In particular, we developed three different online pricing strategies where the Smart is the best in all the metrics we used, when the number of EVs is large, a situation that is closer to the future reality. Moreover, we present the first optimal offline benchmark for the allocation of EVs to charging points which is optimal in terms of charging management and energy demand peek leveling. This centralized mechanism is ideal for benchmarking reasons, but it scales purely, its requirement that full knowledge of car charging demands will be available is unrealistic and, thus, it cannot be used in practice.

Future work will look at predictive models to forecast demand from electric vehicles according to daily usage patterns and ongoing activities in a city. Moreover, we will investigate auction-based mechanisms to allocate charging slots, similar to Gerding et al. [2] to take into account incentive compatibility issues.

\section{ACKNOWLEDGEMENTS}

S. D. Ramchurn was funded by the ORCHID project funded by EPSRC (EP/I011587/1).

\section{REFERENCES}

[1] U. E. I. Administration, "Annual energy review 2011," Tech. Rep. DOE/EIA-0384(2011), 2012.

[2] E. Gerding, S. Stein, D. Zhao, and N. Jennings, "Two-sided online markets for electric vehicle charging," in The Twelfth International Joint Conference on Autonomous Agents and Multi-Agent Systems (AAMAS 2013), 2013.

[3] E. Gerding, V. Robu, S. Stein, D. Parkes, A. Rogers, and N. Jennings, "Online mechanism design for electric vehicle charging," in The Tenth International Joint Conference on Autonomous Agents and Multi-Agent Systems (AAMAS 2011), May 2011, pp. 811-818, event Dates: 2-6 May 2011. [Online]. Available: http://eprints.soton.ac.uk/271907/

[4] S. Stein, E. Gerding, V. Robu, and N. Jennings, "A model-based online mechanism with pre-commitment and its application to electric vehicle charging," in Proc. 11th Int. Conference on Autonomous Agents and Multi-Agent Systems (AAMAS), 2012, pp. 669-676. [Online]. Available: http://eprints.soton.ac.uk/273082/

[5] M. Caramanis and J. Foster, "Management of electric vehicle charging to mitigate renewable generation intermittency and distribution network congestion," in Proceedings of the 48th IEEE Conference on Decision and Control, 2009 held jointly with the 2009 28th Chinese Control Conference. CDC/CCC 2009., dec. 2009, pp. $4717-4722$.

[6] J. Eisner, S. Funke, and S. Storandt, "Optimal route planning for electric vehicles in large networks," in 25th Conf. on Artificial Intelligence (AAAI), 2011.

[7] S. Storandt and S. Funke, "Cruising with a battery-powered vehicle and not getting stranded," in 26th Conf. on Artificial Intelligence (AAAI), 2012.

[8] L. D. B. William J. Mitchel, Christopher E. Borroni-Bird, Reinventing the Automobile. MIT Press, 2010. 